\title{
Critical Time-Window for NO-cGMP-Dependent Long-Term Memory Formation after One-Trial Appetitive Conditioning
}

\author{
Ildikó Kemenes, György Kemenes, Richard J. Andrew, Paul R. Benjamin, and Michael O'Shea \\ Sussex Centre for Neuroscience, School of Biological Sciences, University of Sussex, Falmer, Brighton, United Kingdom, \\ BN1 9QG
}

\begin{abstract}
The nitric oxide (NO)-cGMP signaling pathway is implicated in an increasing number of experimental models of plasticity. Here, in a behavioral analysis using one-trial appetitive associative conditioning, we show that there is an obligatory requirement for this pathway in the formation of long-term memory (LTM). Moreover, we demonstrate that this requirement lasts for a critical period of $\sim 5 \mathrm{hr}$ after training. Specifically, we trained intact specimens of the snail Lymnaea stagnalis in a single conditioning trial using a conditioned stimulus, amylacetate, paired with a salient unconditioned stimulus, sucrose, for feeding. Long-term associative memory induced by a single associative trial was demonstrated at $24 \mathrm{hr}$ and shown to last at least $14 \mathrm{~d}$ after training. Tests for LTM and its dependence on $\mathrm{NO}$ were performed routinely $24 \mathrm{hr}$ after training. The critical period when NO was needed for memory formation was estab-
\end{abstract}

lished by transiently depleting it from the animals at a series of time points after training by the injection of the NO-scavenger 2-phenyl-4,4,5,5-tetramethyl-imidazoline-1-oxyl3-oxide (PTIO). By blocking the activity of NO synthase and soluble guanylyl cyclase enzymes after training, we provided further evidence that LTM formation depends on an intact NO-cGMP pathway. An electrophysiological correlate of LTM was also blocked by PTIO, showing that the dependence of LTM on NO is amenable to analysis at the cellular level in vitro. This represents the first demonstration that associative memory formation after singletrial appetitive classical conditioning is dependent on an intact NO-cGMP signaling pathway.

Key words: one-trial classical conditioning; long-term memory; critical period; nitric oxide; cGMP; Lymnaea

\begin{abstract}
Much evidence has accumulated to show that nitric oxide (NO) is an important neurotransmitter and neuromodulator in both vertebrates and invertebrates (Jacklet, 1997; Müller, 1997; Fedele and Raiteri, 1999). An increasing number of studies also indicate that the NO-cGMP pathway is involved in associative memory formation (Hawkins et al., 1998; Rose, 2000; Schweighofer and Ferriol, 2000), although in some systems its role is controversial (Blokland et al., 1999; Frisch et al., 2000). The notion that NO-dependent processes are important in associative long-term memory (LTM) formation received strong support from recent experiments revealing a link between the NO-cGMP system and activation of the transcription factor cAMP response elementbinding protein (CREB) during long-term potentiation (LTP) in mice (Lu et al., 1999). Also, recent experiments in bees show a role for the NO-cGMP pathway in the prolonged PKA activation required for associative LTM (Müller, 2000). Important though these findings are, they have been obtained in animals in which either cellular analyses are difficult to perform (insects) or the results of such analyses are still often difficult to relate to learning in intact animals (mammals). This has hindered progress toward understanding the role of $\mathrm{NO}$ in terms of altered activity in circuits of identified neurons controlling specific instances of learned behavior.
\end{abstract}

\footnotetext{
Received July 23, 2001; revised Nov. 1, 2001; accepted Nov. 26, 2001.

This work was supported by a United Kingdom Biotechnology and Biological Sciences Research Council Grant. G.K. is supported by the United Kingdom Medical Research Council. We thank Dr. K. Staras for his useful comments on this manuscript.

Correspondence should be addressed to Dr. Ildikó Kemenes, Sussex Centre for Neuroscience, School of Biological Sciences, University of Sussex, Falmer, Brighton, UK, BN1 9QG. E-mail: I.Kemenes@sussex.ac.uk.

Copyright (C) 2002 Society for Neuroscience $0270-6474 / 02 / 221414-12 \$ 15.00 / 0$
}

In the present work, we set out to analyze the dependence of associative behavioral plasticity on NO in an animal in which this is also amenable to cellular analysis. The conditioned response, feeding, and its underlying neuronal circuitry in the snail Lymnaea stagnalis are already an established model system for studying both the cellular mechanisms of learning (Whelan and McCrohan, 1996; Kemenes et al., 1997; Kojima et al., 1997; Staras et al., 1999; Jones et al., 2001) and the function of NO (Moroz et al., 1993; Elphick et al., 1995; Park et al., 1998). In addition, NO synthase (NOS)-containing neurons have been mapped (Elofsson et al., 1993; Moroz et al., 1994; Elphick et al., 1995; Serfözö et al., 1998) and a neuronal NOS mRNA has been cloned, sequenced, and shown to be expressed in the cerebral giant cells (Korneev et al., 1998), modulatory neurons in the Lymnaea feeding system (Benjamin et al., 2000).

That Lymnaea acquires LTM for conditioned feeding after a single trial was first demonstrated by Alexander et al. (1984). This ability, combined with the existing information about both the NO-cGMP pathway and the cellular mechanisms of feeding, made the single-trial paradigm especially attractive. For example, it allows the effects of sharply timed manipulations of the NOcGMP pathway to be analyzed. Here we will demonstrate that an intact NO-cGMP pathway is necessary for LTM and that NO plays a crucial role during the first $5 \mathrm{hr}$ after training. Importantly, we will also show that LTM and its dependence on NO demonstrated at the behavioral level in vivo is amenable to an electrophysiological analysis in vitro.

\section{MATERIALS AND METHODS}

The general aim of the experiments was to test the effect of variously timed interruptions of the NO-cGMP pathway on the formation of LTM after single-trial appetitive classical conditioning. 
Experimental animals. Laboratory-bred stock of Lymnaea stagnalis was obtained from the Free University (Amsterdam, The Netherlands) and kept in groups in large holding tanks containing copper-free water at $18-20^{\circ} \mathrm{C}$ on a $12 \mathrm{hr}$ light/dark cycle. The animals were fed lettuce three times and a vegetable-based fish food (Tetra-Phyll; TETRA Werke, Melle, Germany) twice a week except before starting an experiment, when they were not fed for $5 \mathrm{~d}$.

One-trial conditioning protocol. Appetitive chemical classical conditioning of intact animals was performed using a method based on a previously described single-trial training protocol (Alexander et al., 1984). Twenty-four hours before a conditioning experiment, animals were pretested for feeding responses to a chemical stimulus, amyl-acetate $(0.04 \%$ final concentration), to be used as the conditioned stimulus (CS) during training. The level of response to the CS was established by comparing the snails' feeding rates in water and amyl-acetate. Before the tests, individually isolated snails were left for $15 \mathrm{~min}$ in test dishes containing $90 \mathrm{ml}$ water to acclimatize them to the new environment. Five milliliters of water were then added to the dish, and the number of "rasps" (stereotyped feeding movements consisting of opening of the mouth, rasping with the radula, and closing of the mouth) was counted for the following 2 min, using previously described methods (Kemenes et al., 1986). This established the level of rasping activity for the general disturbance caused by delivering solutions to the dish. At the end of this first $2 \mathrm{~min}$ period, $5 \mathrm{ml}$ of amyl-acetate solution was added, and rasps were counted for a further $2 \mathrm{~min}$ before the snails were transferred into a rinse tank and then back to their home tanks. A score was generated for each animal by subtracting the number of rasps in response to water disturbance from that elicited by amyl-acetate, and this was termed the feeding response to the CS. This procedure (Alexander et al., 1984) helps to reduce the variance of the CS responses within the groups. Various statistical methods (see Results) was used to test whether all groups in a particular experiment were matched for their pretraining feeding responses to water and amyl-acetate and to rule out the possibility that the response to water might have changed during conditioning. This was important because the use of the amyl-acetate minus water response scores to quantify the feeding responses to CS is only valid when it is calculated against stable and group-matched response levels in water, both before and after training.

The pretested snails were assigned to an experimental and a control group, matched for pretraining feeding responses to the CS, and given a single conditioning and control trial, respectively. Before training, each group was placed in a separate tank containing copper-free water and left to acclimatize for at least $15 \mathrm{~min}$. For CS + unconditioned stimulus (US) training, $50 \mathrm{ml}$ of amyl-acetate solution (the CS; $0.04 \%$ final concentration) was poured into $200 \mathrm{ml}$ water in the tank of the experimental group, and this was followed within $15 \mathrm{sec}$ by $50 \mathrm{ml}$ of sucrose solution (the US; $0.67 \%$ final concentration). An explicitly unpaired control group received a CS + water (disturbance control) pairing followed by a water + US pairing $1 \mathrm{hr}$ later. For US alone and CS alone controls, $50 \mathrm{ml}$ of water replaced amyl-acetate and was followed by sucrose, and amyl-acetate was followed by water, respectively. To balance the temporal pattern of disturbance between all four groups, the CS + US, CS alone, and US alone groups were all subjected to a water + water trial $1 \mathrm{hr}$ before training. During the CS + water trial in the unpaired control group and the water + water balancing trial in the other three groups, the animals were allowed to stay in the training dish for 2 min after the application of the second water stimulus. The snails were then lifted out using a plastic mesh, rinsed for $10 \mathrm{~min}$, and finally transferred back to their home tanks containing fresh water where they were kept until the training trial, which was performed $1 \mathrm{hr}$ after the initial trial. The training trial again included an exposure to the CS + US, water + US, or CS + water mixture for 2 min after the application of the second stimulus (US or water) and was followed by the same rinsing and transfer procedures as described for the initial trial. Thus, the duration of all three types of stimulus (CS, US, water) as well as the intertrial and interstimulus intervals were balanced between all four groups used in the experiment. Moreover, the final CS or US concentrations were the same in every trial in all four groups.

All animals were retested, using a blind protocol, for the response to the CS $24 \mathrm{hr}$ after the conditioning or control trial. The post-training tests were performed in exactly the same way as the pretraining ones, and the feeding responses to the CS were also established using the same scoring method (see above). After the tests, the animals were kept in their home tanks on a normal feeding regimen for $8 \mathrm{~d}$ but deprived of food for $5 \mathrm{~d}$ before they were retested at $14 \mathrm{~d}$ after training.
Pharmacological agents and their application. Three types of compounds causing NO depletion, inhibition of NOS, and inhibition of the NO receptor soluble guanylyl cyclase (sGC) were used to interfere with the NO-cGMP signaling pathway (see Fig. 1).

A specific and stable radical scavenger of NO (Akaike et al., 1993), 2-phenyl-4,4,5,5-tetramethyl-imidazoline-1-oxyl 3-oxide (PTIO) (SigmaAldrich, Poole, UK), was used at a $1 \mathrm{~mm}$ estimated final concentration to deplete NO from the animals. Previous work showed that this concentration was sufficient to block NO-dependent activation of the fictive feeding response in Lymnaea (Kobayashi et al., 2000).

An inhibitor of the NOS enzyme, $N^{\mathrm{G}}$-Nitro-L-arginine-methyl ester hydrochloride (L-NAME) (Alexis Biochemicals, Bingham, UK) was used at a $25 \mathrm{~mm}$ estimated final concentration, and $N^{\mathrm{G}}$-Nitro-D-argininemethyl ester hydrochloride (D-NAME; $25 \mathrm{mM}$ ) was used as the ineffective isomer. In this concentration range, L-NAME, but not D-NAME, was shown previously to block NO-mediated network oscillations in olfactory interneurons in Limax (Gelperin, 1994) and the NO-dependent activation of fictive feeding by sucrose in Lymnaea (Elphick et al., 1995). In molluscs, high concentrations of L-NAME are required to block NOS activity because of the presence of high intracellular concentrations $(\sim 6$ $\mathrm{mM}$ ) of L-arginine in NOS-containing neurons (Floyd et al., 1998).

A potent and selective inhibitor of NO-sensitive sGC, $1 \mathrm{H}-(1,2,4)$ oxidazole (4,3-a) quinoxalin-1-one (ODQ) (Alexis Biochemicals) was used at a $10 \mu \mathrm{M}$ estimated final concentration. The effectiveness of ODQ in the nanomolar to micromolar concentration range has been demonstrated both in mammals [rats (Garthwaite et al., 1995)] and in molluscs [Aplysia (Koh and Jacklet, 1999, 2001; Lewin and Walters, 1999) and Helisoma (Van Wagenen and Rehder, 1999)]. Because a Lymnaea sGC has been identified and shown to have a substantial homology with the mammalian sGC (Ogunshola et al., 1995), and also because Helisoma and Lymnaea are very closely related species, it was reasonable to assume that any behavioral effect of ODQ would be caused by an inhibition of the Lymnaea sGC enzyme.

All solutions were prepared in snail physiological saline (Benjamin and Winlow, 1981), except in the case of ODQ, which was first dissolved in $100 \%$ DMSO and then dissolved in saline before the experiment $(0.1 \%$ final DMSO concentration). Injection of pharmacological agents and their controls was performed using Hamilton syringes with fine needles (26 gauge; Aldrich, Milwaukee, WI). Because there is no blood-brain barrier in the snail (Sattelle and Lane, 1972), chemical agents injected into the hemolymph will have direct access to the CNS. One hundred microliters of a particular solution were injected into the body cavity of each snail. Because the estimated volume of the hemolymph is $\sim 1 \mathrm{ml}$, the estimated final concentration of the drugs was calculated as one-tenth of the injected concentration.

Effects of PTIO, L-NAME, and $O D Q$ on unconditioned feeding behavior. To our knowledge, PTIO has not been used before in learning studies, and therefore we had to develop the appropriate protocol for its application, also taking into account its potential effects on the unconditioned behavior. This was particularly important because NO was known to be involved in the sensory pathway involved in sucrose-activated unconditioned feeding behavior in Lymnaea (Elphick et al., 1995). Because sucrose is the US in the training experiment, it was essential to know when normal activation of feeding by sucrose resumed after PTIO injection. Animals were pretested for sucrose response (see Fig. $2 A,-60$ min) using the standard procedure (identical to the pretest for amylacetate) to normalize for disturbance (i.e., 2 min water +2 min sucrose). The pretested snails were then divided into two matched groups. One hour later, PTIO (1 mM final concentration) was injected into the snails in one of the groups $(n=9)$, whereas snails in the other group $(n=9)$ received saline injection (see Fig. $2 A, 0 \mathrm{~min}$ ). All snails in both groups were retested for their feeding response to sucrose $30 \mathrm{~min}, 1 \mathrm{hr}$, and $2 \mathrm{hr}$ after injection (see Fig. 2A). The saline-injected group was used to obtain information on the time-dependent effect of injection with the vehicle alone and for statistical comparisons with the effects of PTIO injection at the same time points. Use of a vehicle-control group also allowed the snails from the two different groups to be tested using a blind protocol.

A two-way ANOVA with repeated measures was performed on both before and after injection feeding rates in both groups. This established the existence of a significant interaction between drug treatment and time in the whole experiment $\left(F_{3,70}=11.9 ; p<0.001\right)$. However, the feeding response in the saline- and PTIO-injected groups showed very different profiles as time progressed after injection. In the saline-injected group, no significant changes were detected over time, whereas within the 
PTIO-injected group a significant source of difference was detected $\left(F_{3,34}=14.3 ; p<0.001\right)$. We found that at $30 \mathrm{~min}$ after injection, the feeding response of PTIO-injected snails [2.6 $\pm 2.7(\mathrm{SE}) \mathrm{rasps} / 2 \mathrm{~min}]$ was significantly weaker compared with both saline-injected control levels $(24.2 \pm 1.9 \mathrm{rasps} / 2 \mathrm{~min} ; p<0.001)$ and their own preinjection levels $(18.1 \pm 1.9 \mathrm{rasps} / 2 \mathrm{~min} ; p<0.003)$. In contrast, 1 and $2 \mathrm{hr}$ after injection there was no significant difference between either the post-injection feeding responses of PTIO and saline-injected snails or between preinjection and post-injection feeding responses of PTIO-injected snails (see Fig. $2 B$ ). This experiment showed that depletion of NO inhibited the feeding response for $>30 \mathrm{~min}$ but $<1 \mathrm{hr}$ after PTIO injection. This information was used to infer the duration of PTIO effect on the NO-dependent component of learning.

Using a similar protocol, a significant time-dependent effect of drug treatment on the unconditioned feeding response was also established in an L-NAME injection experiment $\left(F_{3,86}=6.4 ; p<0.001\right)$. However, unlike PTIO, L-NAME still had a significant effect on the feeding response $1 \mathrm{hr}$ after the injection $(18.7 \pm 2.7 \mathrm{rasps} / 2 \mathrm{~min})$, compared both with D-NAME-injected controls $(27.7 \pm 1 \mathrm{rasps} / 2 \mathrm{~min} ; p<0.01)$ and with preinjection response levels $(30.2 \pm 1.5 \mathrm{rasps} / 2 \mathrm{~min} ; p<0.01)$. At 3 hr after injection, L-NAME still had a weak but significant inhibitory effect on feeding $(26.3 \pm 1.3 \mathrm{rasps} / 2 \mathrm{~min})$ when compared with preinjection levels $(p<0.001)$ but not with D-NAME controls. The feeding response returned to a normal level $(29.6 \pm 1.6 \mathrm{rasps} / 2 \mathrm{~min})$ only $6 \mathrm{hr}$ after injection.

The same tests were performed before and after ODQ injection, and similarly strong interactions between drug treatment and time were found as with PTIO and L-NAME $\left(F_{4,118}=12.7 ; p<0.001\right)$. The effect of ODQ was found to be even longer lasting than in the case of L-NAME. Although the feeding response to sucrose was normal $24 \mathrm{hr}$ after injection $(27.9 \pm 1.1 \mathrm{rasps} / 2 \mathrm{~min})$ and there was a steady recovery during the first few hours, it was still significantly lower even at $6 \mathrm{hr}$ after injection $(20.7 \pm 1.3 \mathrm{rasps} / 2 \mathrm{~min})$ than the response level in saline + DMSOinjected controls $(29.8 \pm 1.0 \mathrm{rasps} / 2 \mathrm{~min} ; p<0.001)$ or the preinjection response level $(29.4 \pm 1.5 \mathrm{rasps} / 2 \mathrm{~min} ; p<0.001)$.

Effects of NO depletion on LTM. The first aim was to establish the effect of NO depletion shortly after the acquisition phase of learning on the formation of long-term associative memory. To achieve this, we investigated the effect of PTIO on the conditioned response after injecting it into a group of CS + US animals 10 min after training.

The experiment was performed as follows. A group of animals were conditioned with a single pairing of the amyl-acetate CS with the sucrose US, whereas an unpaired control group received the CS and US separated by a $1 \mathrm{hr}$ interval, as described earlier. This is the most important control in this type of experiment, because it includes the use of both CS and US. The lack of a conditioned response in other types of controls, such as CS alone and US alone, has been demonstrated by the earlier experiments (Alexander et al., 1984) and confirmed in the present work (see Results). Subsequently, half of the conditioned and half of the unpaired control animals were injected with PTIO 10 min after the trial, whereas the other half of each group received saline injections (vehicle control). The effects of PTIO and saline on the response to the CS were tested in a blind protocol $24 \mathrm{hr}$ after training in all four groups used in this experiment.

The time course of the effect of depletion of NO on LTM was initially determined in experiments in which a large number of conditioned animals were injected with PTIO at seven different time points after a single training trial. Before training, animals were divided into seven groups, one for each time point, matched for pretraining feeding response levels to amyl-acetate. In the first $6 \mathrm{hr}$ after training, $1 \mathrm{hr}$ time intervals were chosen for PTIO injection. This was based on the experiment investigating the temporary NO-depleting effects of PTIO in intact Lymnaea (see Fig. 2). If the duration of action of PTIO after injection is no more than $1 \mathrm{hr}$, as suggested by its direct action on feeding, then an offset of sensitivity in this time course would imply action up to approximately the next time point. All groups were retested for the response to the CS in a blind protocol $24 \mathrm{hr}$ after training. Further experiments were performed using both conditioned and unpaired control groups to confirm that NO depletion at critical time points after training was interfering with the formation of associative LTM.

Effects of NOS and $s G C$ inhibition on LTM. The drugs L-NAME and ODQ were also injected 10 min after single-trial conditioning, and their effects on LTM were tested at $24 \mathrm{hr}$ after training, as described previously for PTIO. These two drugs were not suitable for the same detailed time course analysis as PTIO because of their more prolonged behavioral effects, described earlier in this section.

Statistical analysis. All statistical analysis was performed using parametric methods. Pretraining or post-training/injection comparisons between multiple groups were made using a one-way ANOVA. The timedependent interactions between two variables (training/control procedure and drug/vehicle injection) were established using a two-way ANOVA with repeated measures (SPSS, General Linear Model, Repeated Measures Procedure), and the groups significantly affected by the interacting factors were identified by a two-way ANOVA (SPSS statistical package, General Linear Model). Where appropriate, ANOVAs were followed by multiple post hoc tests [SPSS, Tukey's honestly significant difference (HSD) test] to establish differences between pairs of groups. Feeding responses in the same group before and after training or injection were compared using paired $t$ tests. When only two different groups were compared, pairwise between-group comparisons were made using unpaired $t$ tests.

LTM in vitro. As a prelude to further analysis at the cellular level, we demonstrate that electrophysiological correlates of the effects of NO depletion on LTM formation in vivo can be recorded in vitro. After establishing the effect of PTIO and saline injection in both conditioned and unpaired control animals, animals were conditioned with a single $\mathrm{CS}+$ US pairing and then split into two groups, matched for pretraining response to the CS. Ten minutes after conditioning, snails in one of the groups were injected with PTIO, and the others were injected with saline. Intact animals were first tested blind for LTM $24 \mathrm{hr}$ after training. Animals chosen randomly from both the PTIO- and saline-injected groups were dissected to provide semi-intact lip-CNS preparations in which to measure the electrophysiological effects in selected identified neurons of the CS applied to the lip. Specifically, an identified phasic modulatory interneuron (CV1a) and an identified motoneuron (B3) of the feeding network [for a recent overview, see Benjamin et al. (2000)] were used to monitor the occurrence of fictive feeding cycles after application of amyl-acetate to the lips. The type of preparation and electrophysiological techniques used in this work have been described recently in detail (Kemenes et al., 2001).

\section{RESULTS}

\section{LTM after single-trial appetitive classical conditioning}

Robust and highly significant LTM lasting for at least 2 weeks was achieved after a single pairing of the amyl-acetate CS and sucrose US in hungry snails. This was demonstrated with various statistical methods using the pretraining and post-training feeding response data obtained in water and amyl-acetate and also using the feeding response to the CS data.

All four groups used in this experiment showed uniformly low pretraining feeding rates in response to water (CS + US, $2.3 \pm$ $0.9 \mathrm{rasps} / 2 \mathrm{~min}$; unpaired, $3.1 \pm 1.2 \mathrm{rasps} / 2 \mathrm{~min}$; CS alone, $2.8 \pm$ $1.4 \mathrm{rasps} / 2 \mathrm{~min}$; US alone, $3.7 \pm 1.5 \mathrm{rasps} / 2 \mathrm{~min}$; ANOVA, $F_{3,36}=$ $0.22 ; p=0.90)$. The pretraining responses in the presence of amyl-acetate were also uniformly low in all four groups (CS + US, $4.2 \pm 1.8 \mathrm{rasps} / 2 \mathrm{~min}$; Unpaired, $4.6 \pm 1.2 \mathrm{rasps} / 2 \mathrm{~min}$; CS alone, $4.5 \pm 1.4 \mathrm{rasps} / 2 \mathrm{~min}$; US alone, $5.1 \pm 1.5 \mathrm{rasps} / 2 \mathrm{~min}$; ANOVA, $F_{3,36}=0.09 ; p=0.96$ ). Moreover, when all the pretraining water and amyl-acetate feeding response rates were subjected to an ANOVA together, this did not reveal an overall significant difference either $\left(F_{7,72}=0.43 ; p=0.87\right)$. Thus, the difference scores we used to determine the feeding response to the CS before training were low, with no overall statistical difference between the four groups (see Fig. 3Bi; ANOVA, $F_{3,36}=$ $0.06 ; p=0.98)$.

Comparing all before- and after-training feeding responses to the CS data in all four groups by an ANOVA with repeated measures, a significant overall difference was detected both within $\left(F_{2,72}=7.9 ; p<0.01\right)$ and between the groups $\left(F_{3,36}=26.5 ; p<\right.$ 0.0001 ). Detailed post hoc tests revealed that the only sources of the between-group differences were the significant differences between the post-training responses of the conditioned group and 
those of each of the three control groups, both at $24 \mathrm{hr}$ and $14 \mathrm{~d}$ after training. When tested $24 \mathrm{hr}$ after training (see Fig. 3Bii), the CS + US conditioned animals $(n=10)$ showed feeding responses to the $\mathrm{CS}(15.7 \pm 1.6 \mathrm{rasps} / 2 \mathrm{~min})$ that were significantly stronger than post-training responses to the CS in explicitly unpaired $(1.8 \pm 1.2 \mathrm{rasps} / 2 \mathrm{~min} ; n=10)$, CS alone $(2.0 \pm 1.1 \mathrm{rasps} / 2 \mathrm{~min}$; $n=10)$, or US alone controls $(1.6 \pm 1.8 \mathrm{rasps} / 2 \mathrm{~min} ; n=10)$ (Tukey's HSD tests; $p<0.001$ ). Significant differences between the conditioned group and each of the control groups were retained $14 \mathrm{~d}$ after the training (Tukey's HSD tests; $p<0.003$ ) (see Fig. 3Biii). Moreover, in both the $24 \mathrm{hr}$ and the $14 \mathrm{~d}$ tests, only the conditioned animals showed feeding responses to the CS that were significantly higher than their own pretraining scores (1.9 \pm 0.9 rasps $/ 2$ min; paired $t$ tests; $p<0.001)$, demonstrating that the significant overall within-group differences revealed by the repeated-measures ANOVA were caused by pretraining to post-training changes in the CS + US group only.

The low feeding responses to the CS seen in the control groups at both $24 \mathrm{hr}$ and $14 \mathrm{~d}$ after training arose from small differences between low post-training feeding rates, both in the presence of water and in the presence of amyl-acetate. Thus, at $24 \mathrm{hr}$ after training, the unpaired control group showed $3.2 \pm 1.3 \mathrm{rasps} / 2 \mathrm{~min}$ in water and $5.0 \pm 3.3 \mathrm{rasps} / 2 \mathrm{~min}$ in amyl-acetate. When retested $14 \mathrm{~d}$ after training, the same group showed $1.0 \pm 2.3 \mathrm{rasps} / 2 \mathrm{~min}$ in water and $2.7 \pm 2.2 \mathrm{rasps} / 2 \mathrm{~min}$ in amyl-acetate. At the same two post-training time points, the CS alone control group showed $3.6 \pm 2.4$ and $3.7 \pm 2.4 \mathrm{rasps} / 2 \mathrm{~min}$ in water and $5.1 \pm 3.4$ and $5.6 \pm 3.9 \mathrm{rasps} / 2 \mathrm{~min}$ in amyl-acetate, whereas the US alone control group showed $2.3 \pm 1.6$ and $2.9 \pm 1.8 \mathrm{rasps} / 2 \mathrm{~min}$ in water and $3.9 \pm 2.6$ and $5.0 \pm 3.8 \mathrm{rasps} / 2 \mathrm{~min}$ in amyl-acetate. In the control groups, neither the response to water nor the response to amyl-acetate increased between the pretraining and the two posttraining test times (doubly multivariate ANOVA with repeated measures; $F_{4,108}=0.18 ; p=0.95$ ). On the other hand, the high post-training feeding response to the CS scores in the conditioned group both at $24 \mathrm{hr}$ and $14 \mathrm{~d}$ after training were attributable to large differences between low feeding rates in water and much higher feeding rates in amyl-acetate. Thus, at $24 \mathrm{hr}$ and $14 \mathrm{~d}$ after training, the responses of the conditioned group in water were $3.8 \pm 2.3$ and $1.2 \pm 2.1 \mathrm{rasps} / 2 \mathrm{~min}$, respectively. These did not differ significantly from the pretraining response of the same group in water (ANOVA with repeated-measures; $F_{2,72}=0.95$; $p=0.39)$. In contrast, the post-training responses of the conditioned group in amyl-acetate $(19.5 \pm 6.7$ and $15.6 \pm 5.3 \mathrm{rasps} / 2$ min) showed significant increases over the pretraining response level $\left(F_{2,72}=6.2 ; p<0.003\right)$. As a final test to validate the use of amyl-acetate minus water feeding response scores to quantify the feeding response to the $\mathrm{CS}$ in all subsequent experiments, we performed a doubly multivariate ANOVA with repeated measures on all four groups used in this experiment. This revealed a significant overall within-group multivariate effect $\left(F_{4,144}=4.2\right.$; $p<0.003$ ) and a significant interaction of within-group (time of test) and between-group (type of training) effects $\left(F_{12,144}=3.0\right.$; $p<0.001)$. Importantly, the same analysis also detected a significant overall difference in the amyl-acetate responses of the four groups $\left(F_{3,36}=12.9 ; p<0.0001\right)$, whereas no overall differences were revealed in the water responses of the same groups $\left(F_{3,36}=\right.$ $0.69 ; p=0.56$ ). Post hoc analyses (Tukey's HSD tests) confirmed that the only sources of the overall significant difference in the amyl-acetate responses were the significant $(p<0.0001)$ pairwise differences between the post-training amyl-acetate feeding re- sponses of the CS + US trained group and those of each of the three control groups.

The results of the above experiments therefore confirmed and significantly extended the earlier findings of Alexander et al. (1984) in the population of animals used in our subsequent behavioral pharmacological experiments.

\section{Depletion of NO prevents LTM formation}

The effect of NO depletion on the formation of LTM, tested $24 \mathrm{hr}$ after training, was first examined in experiments in which PTIO was injected into conditioned and unpaired control animals 10 min after the trial (see Fig. 4A). For comparison, the effect of injection with the vehicle (saline) alone was also examined in conditioned and unpaired control animals (see Fig. 4A). All groups in this experiment were matched for their pretraining feeding responses to the CS (see Fig. $4 \mathrm{Bi}$ ). This was confirmed by an ANOVA that also showed that the groups were matched for their pretraining feeding rates in the presence of both water and amyl-acetate. The mean feeding response levels found in the four groups ranged from 1.8 to $2.5 \mathrm{rasps} / 2 \mathrm{~min}$ in water and 2.7 to 3.5 rasps $/ 2 \mathrm{~min}$ in amyl-acetate, with no significant overall differences (ANOVA) between the groups. Finally, using an ANOVA with repeated measures we confirmed that the feeding responses to water remained unchanged in all four groups between the pretraining and post-training tests (the mean post-training responses ranged from 1.9 to $2.5 \mathrm{rasps} / 2 \mathrm{~min}$ ). Thus, any pretraining to post-training changes in feeding responses to the CS could only be attributed to changes in the feeding response levels in the presence of amyl-acetate.

To explore the interactions between drug/vehicle injection and conditioning/control procedures, an ANOVA with repeated measures was performed using pretraining (see Fig. 4Bi) and posttraining (see Fig. 4Bii) feeding response to CS data. This revealed highly significant effects for both training and drug treatment $\left(F_{1,71}=22.3\right.$ and $12.9 ; p=0.001$ for both $)$ and a highly significant interaction between these two factors $\left(F_{1,71}=12.8\right.$; $p=0.001)$. To identify groups significantly affected by this strong interaction, a two-way ANOVA was performed. This revealed at least one source of significant difference between the posttraining responses of the four groups subjected to different combinations of training/control and drug/vehicle treatment $\left(F_{3,74}=\right.$ $17.2 ; p<0.001)$. Conditioned snails injected with saline $(n=20)$ showed high levels of responses to the CS at test $(15.1 \pm 2.0$ rasps/2 $\mathrm{min}$ ) that were significantly greater than the responses $(1.7 \pm 1.5 \mathrm{rasps} / 2 \mathrm{~min})$ of the unpaired controls injected with saline $(n=19)$ (Tukey's HSD tests; $p<0.002)$ (see Fig. 4Bii). The CS-evoked feeding responses of saline-injected conditioned animals were also significantly higher than their own pretraining scores $(p<0.0001)$ (see Fig. $4 B i, B i i)$. In contrast, conditioned snails injected with PTIO 10 min after training $(n=20)$ showed response levels $(3.8 \pm 1.2 \mathrm{rasps} / 2 \mathrm{~min})$ to the CS $24 \mathrm{hr}$ later that were not significantly different from the responses $(1.9 \pm 1.3$ rasps/2 min) of unpaired control snails $(n=19)$ also injected with PTIO (see Fig. 4Bii), or from their own pretraining response levels $(1.0 \pm 0.8 \mathrm{rasps} / 2 \mathrm{~min})$. Taken together, these results showed that although in the animals used in this experiment a memory trace could be formed and was resistant to injection with the vehicle shortly after training, its formation could be prevented by injection of PTIO at the same early time point. This experiment therefore indicated that shortly after conditioning, NO was needed for the formation of associative LTM. 


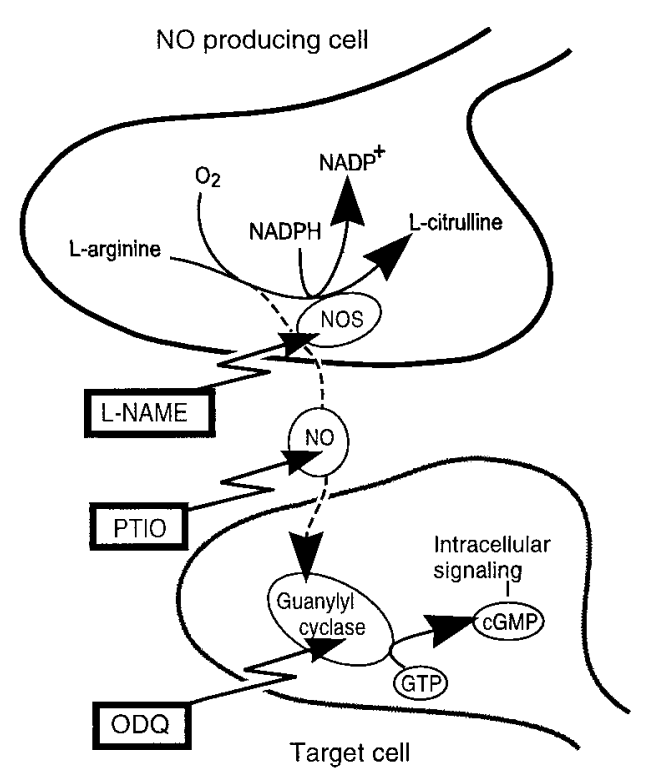

Figure 1. Components of the NO-cGMP signaling pathway and the drugs that were used to target them in the present experiments. PTIO, a nitric oxide $(N O)$ scavenger, was used to temporarily deplete NO from intact Lymnaea. L-NAME was used as an inhibitor of the nitric oxide synthase (NOS) enzyme, and ODQ was used as an inhibitor of the soluble guanylyl cyclase enzyme (for details, see Materials and Methods).

\section{Blocking NO and cGMP synthesis prevents LTM formation}

To investigate whether the early requirement for $\mathrm{NO}$ after training depends on the NO-cGMP signaling pathway, we inhibited NOS and sGC, the two principal enzymes of the pathway. L-NAME was used to block the production of NO by NOS and ODQ to prevent the production of cGMP by the NO-activated sGC, respectively (Fig. 1). Four groups of animals, matched for their mean feeding responses to the CS (see Fig. 4Ci, Di) were used for each of these experiments. An ANOVA confirmed that the same groups were also matched for their pretraining responses to water and amyl-acetate. The mean responses ranged from 1.7 to $3.1 \mathrm{rasps} / 2 \mathrm{~min}$ in water and from 4.7 to $6.8 \mathrm{rasps} / 2$ min in amyl-acetate, with no significant overall differences (ANOVA) between the groups. An ANOVA with repeated measures also confirmed that the mean post-training feeding responses to water (ranging from 0.6 to $3.5 \mathrm{rasps} / 2 \mathrm{~min}$ ) remained unchanged between the pretraining and post-training tests.

An ANOVA with repeated measures in the NOS inhibition experiment demonstrated highly significant effects for both training and drug treatment $\left(F_{1,77}=11.3\right.$ and $7.9 ; p<0.001$ and 0.006 , respectively) and a significant interaction between these two factors $\left(F_{1,77}=11.5 ; p<0.001\right)$. A two-way ANOVA performed on the post-training data $\left(F_{3,77}=18.7 ; p<0.0001\right)$ and followed by post hoc pairwise comparisons showed that there was a significant difference between trained $(12.1 \pm 1.7 \mathrm{rasps} / 2 \mathrm{~min} ; n=21)$ and control snails $(1.7 \pm 1.2 \mathrm{rasps} / 2 \mathrm{~min} ; n=20 ; p<0.001)$, both injected with the inactive form of the NOS inhibitor, D-NAME (see Fig. 4Cii). This was further confirmed by the finding that trained and D-NAME-injected animals showed significantly increased feeding responses to the CS compared with pretraining response levels $(p<0.0002)$ (see Fig. $4 \mathrm{Ci}, \mathrm{Cii})$. This showed both that the animals could be conditioned and that D-NAME was ineffective in blocking memory formation. In contrast, application of the active NOS inhibitor L-NAME 10 min after conditioning completely blocked the formation of memory (see Fig. 4Cii). Trained and L-NAME-injected animals $(n=21)$ responded to the CS $24 \mathrm{hr}$ after the conditioning at a similarly low level (1.7 \pm $1.0 \mathrm{rasps} / 2 \mathrm{~min})$ as before training $(2.8 \pm 1.4 \mathrm{rasps} / 2 \mathrm{~min})$ and as L-NAME-injected unpaired controls after training $(1.4 \pm 0.9$ rasps/2 min; $n=19$ ) (see Fig. 4Cii).

A similar experiment was performed with the inhibition of the NO-sensitive soluble guanylyl cyclase enzyme by ODQ (see Fig. $4 D$ ), in which again significant effects for both training and drug treatment $\left(F_{1,69}=6.9\right.$ and $11.2 ; p<0.01$ and $p<0.001$, respectively) and significant interactions between these factors were found $\left(F_{1,69}=61.7 ; p<0.001\right)$. There was no significant difference between conditioned $(n=17)$ and unpaired control snails $(n=17)$ injected with ODQ 10 min after training (see Fig. 4Dii), indicating that inhibiting sGC impaired memory formation. Similar to the PTIO and L-NAME experiments, a source of significant difference between the groups was revealed by a two-way ANOVA $\left(F_{3,69}=10.7 ; p<0.001\right)$. Feeding responses to the CS $(12.0 \pm 2.1 \mathrm{rasps} / 2 \mathrm{~min})$ in vehicle (saline $+0.1 \% \mathrm{DMSO})$ injected conditioned animals $(n=19)$ were significantly higher than in vehicle-injected unpaired controls $(n=20 ; 3.6 \pm 0.9$ rasps/2 min; Tukey's HSD test; $p<0.001)$ or before training $(3.8 \pm 1.3 \mathrm{rasps} / 2 \mathrm{~min} ; p<0.001)$, indicating that the vehicle alone did not affect memory formation (see Fig. 4Di,Dii).

By blocking the NO synthase and soluble guanylyl cyclase enzymes in the above experiments, we provided further evidence that a deficit of the NO-cGMP pathway shortly after learning results in memory impairment.

\section{Distinct time window for the effect of NO depletion on LTM formation}

For an initial determination of the duration of the requirement for NO in the formation of LTM, we injected snails with PTIO at different time intervals after training. PTIO was selected for this experiment because its effects are short-lived as demonstrated by experiments showing that it interferes with feeding for $<1 \mathrm{hr}$ (Fig. 2B). PTIO can therefore be used for accurately timed, short-term interruptions of NO-dependent processes. Both L-NAME and ODQ were unsuitable for this type of experiment because their behavioral effects are not reversible in the short term (see Materials and Methods).

PTIO was injected into different groups of conditioned animals at seven different time points after training [1 hr $(n=13) ; 2 \mathrm{hr}$ $(n=12) ; 3 \mathrm{hr}(n=13) ; 4 \mathrm{hr}(n=12) ; 5 \mathrm{hr}(n=12) ; 6 \mathrm{hr}(n=$ $13)$; and $21 \mathrm{hr}(n=12)$; see Fig. $5 A i]$. The $3 \mathrm{hr}$ interval between depletion of $\mathrm{NO}$ and test at $24 \mathrm{hr}$ after training in the last group was well outside the time interval during which PTIO-induced NO depletion affected the feeding response (up to $1 \mathrm{hr}$ ) (Fig. 2B). All seven experimental groups were derived from a single large group of animals subjected to a single conditioning trial at the same time. The groups were matched for their pretraining feeding responses to the $\mathrm{CS}$ (see Fig. 5Aii, white bars). The same groups were also found to be matched (ANOVA) for mean pretraining responses to water (ranging from 1.2 to 2.9 rasps/2 min) and amyl-acetate (ranging from 4.7 to $6.5 \mathrm{rasps} / 2 \mathrm{~min}$ ). It was also confirmed (ANOVA with repeated measures) that all seven groups remained matched for their post-training responses to water, which ranged from 1.1 to $3.4 \mathrm{rasps} / 2 \mathrm{~min}$.

To establish whether the seven groups injected at different post-training time points with PTIO showed differences in their responses to the CS, we first performed a two-by-seven ANOVA with repeated measures. This analysis provided statistical com- 
A

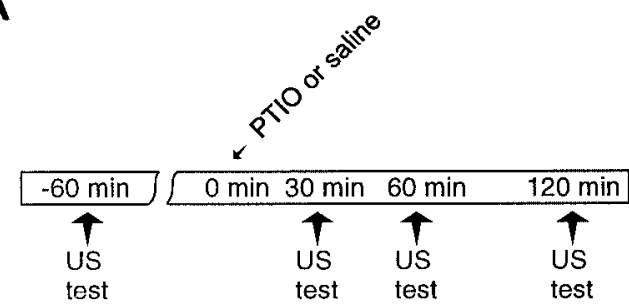

B

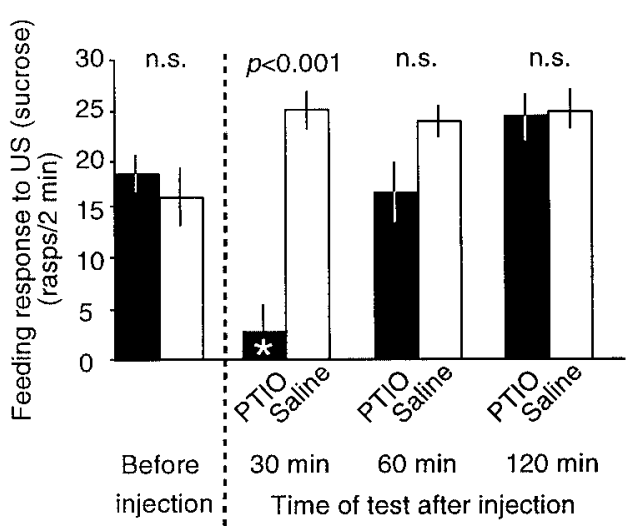

Figure 2. Depletion of NO by PTIO (1 mM final concentration) temporarily impairs the feeding response of Lymnaea to sucrose. $A$, Snails were tested for preinjection feeding responses to sucrose (US test, $-60 \mathrm{~min}$ ) and retested at three different time points $(30 \mathrm{~min}, 60 \mathrm{~min}$, and $120 \mathrm{~min})$ after injection (at $0 \mathrm{~min}$ ) with PTIO or saline (vehicle control). $B$, PTIOinjected snails (PTIO, black bars), matched for preinjection response levels (Before injection) with saline-injected snails (Saline, white bars), show a significant impairment of the feeding response to sucrose at $30 \mathrm{~min}$ after injection. There is a full recovery of the feeding response by $60 \mathrm{~min}$ after injection with PTIO. Data are shown as means \pm SE. Asterisk indicates a significant difference $(p<0.003)$ between postinjection and preinjection response levels.

parisons of the feeding responses to the CS both between the two test time points (before and after training) and the seven different PTIO injection time points. It identified both an overall significant difference between pretraining and post-training response levels and a significant interaction between the effect of training and the timing of PTIO injection $\left(F_{1,80}=6.9, p<0.02\right.$, and $F_{6,80}=3.0, p<0.0001$, respectively). The results of the above ANOVA justified the use of paired $t$ tests on pretraining versus post-training responses within each group to identify sources contributing to the overall pretraining-post differences. The use of paired comparisons to assess the ability of the seven different PTIO-injected groups to form a memory trace was further justified by the results of our previous statistical comparisons between conditioned and unpaired control snails. These demonstrated that only the conditioned groups that showed a significant increase in their CS response levels relative to unpaired controls showed a significant increase in their post-training versus pretraining responses to the CS, established by paired $t$ tests (Figs. 3, 4). In the time course experiment shown in Figure $5 A$, paired comparisons showed no differences between pretraining and post-training response levels to amyl-acetate at any of the first five time points, indicating that LTM was not established in the absence of NO during this period (Fig. 5Aii). The apparent enhanced feeding response to amyl-acetate (CS) in the animals injected at $5 \mathrm{hr}$ after training was not significant when analyzed statistically. At $6 \mathrm{hr}$ after training, PTIO injection did not prevent LTM, as indicated by the statistically significant difference between the post-training and pretraining responses to the CS (after training, $10.0 \pm 3.0$ rasps $/ 2 \mathrm{~min}$, versus before training, $3.4 \pm 1.7 \mathrm{rasps} / 2 \mathrm{~min} ; p<$ 0.02). As expected, injection of PTIO at $21 \mathrm{hr}$ after training also failed to block memory formation. These results suggested that for LTM formation there was a strong dependence on NO for 4 hr after training. This dependence started to decline at some time between 4 and $5 \mathrm{hr}$ after training, and the decline was complete between 5 and $6 \mathrm{hr}$.

The time dependence of the need for NO in memory formation was further confirmed by an examination of the between-groups component (injection timing) of the same repeated-measures ANOVA procedure that established overall significant effects of training within the groups. This revealed no overall difference between the CS responses of the seven groups before conditioning. In contrast, an overall significant difference was identified between the CS responses of the seven different groups after training $\left(F_{6,80}=3.4 ; p<0.005\right)$. Post hoc tests showed that the conditioned responses of the two groups injected with PTIO 6 and $21 \mathrm{hr}$ after training were significantly stronger than the responses seen in the four groups injected $<5 \mathrm{hr}$ after training (Tukey's HSD tests; $p<0.0001$ to 0.02 ). The $5 \mathrm{hr}$ post-training injection group was found to occupy an intermediate position. It was significantly different only from the $1 \mathrm{hr}(p<0.002)$ but not from the 2, 3, or $4 \mathrm{hr}$ post-training injection groups. Neither did it differ significantly from the 6 and $21 \mathrm{hr}$ post-training injection groups.

Taken together, these results strongly supported the conclusion that LTM becomes independent of NO at $\sim 5 \mathrm{hr}$ after training.

We repeated the above experiment at the two time points (4 and $6 \mathrm{hr}$ ) that flank the critical $5 \mathrm{hr}$ time point when it first appears that NO depletion no longer fully prevents LTM. Importantly, in this experiment we used explicitly unpaired control animals to establish whether the higher response to the CS seen at $6 \mathrm{hr}$ was indeed attributable to associative learning. The procedures involved in this experiment are shown in Figure 5Bi. Animals were pretested for the response to CS and divided into two groups, matched for pretraining feeding responses to the CS (Fig. 5Bi,Bii). The feeding responses to water and amyl-acetate (ranging from 1.2 to $2.5 \mathrm{rasps} / 2 \mathrm{~min}$ and 1.5 to $3.5 \mathrm{rasps} / 2 \mathrm{~min}$, respectively) were also not different between the groups (ANOVA). In one group, animals were subjected to one-trial conditioning and in the other to unpaired control training, as described previously (Fig. 4A). Both the conditioned and unpaired control animals were then subdivided into two matched subgroups for injection at two different time points after conditioning. Animals in one of the conditioned and one of the unpaired groups were injected with PTIO $4 \mathrm{hr}$ after training (Fig. $5 B i$, Group 1), the last time point after training when injection of PTIO caused a complete block of memory formation (Fig. 5Aii). The second pair of conditioned and control groups were injected with PTIO 6 hr after training (Fig. 5Bi, Group 2), a time point at which PTIO was first shown to be ineffective (Fig. 5Aii). All animals were retested for water and amyl-acetate (the CS) in a blind protocol $24 \mathrm{hr}$ after training, and an ANOVA with repeated measures again detected no changes in the water response rates between the pretraining and post-training tests.

Conditioned animals $(n=19)$ injected with PTIO $4 \mathrm{hr}$ after training showed no significant differences in their feeding response to the CS $(0.1 \pm 0.8 \mathrm{rasps} / 2 \mathrm{~min})$ compared with unpaired controls $(0.5 \pm 1.1 \mathrm{rasps} / 2 \mathrm{~min} ; n=19)$ or pretraining response 
A

\section{Conditioned}

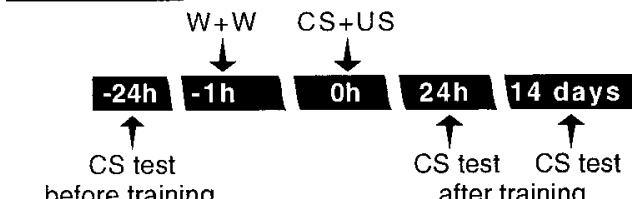

before training

Unpaired control
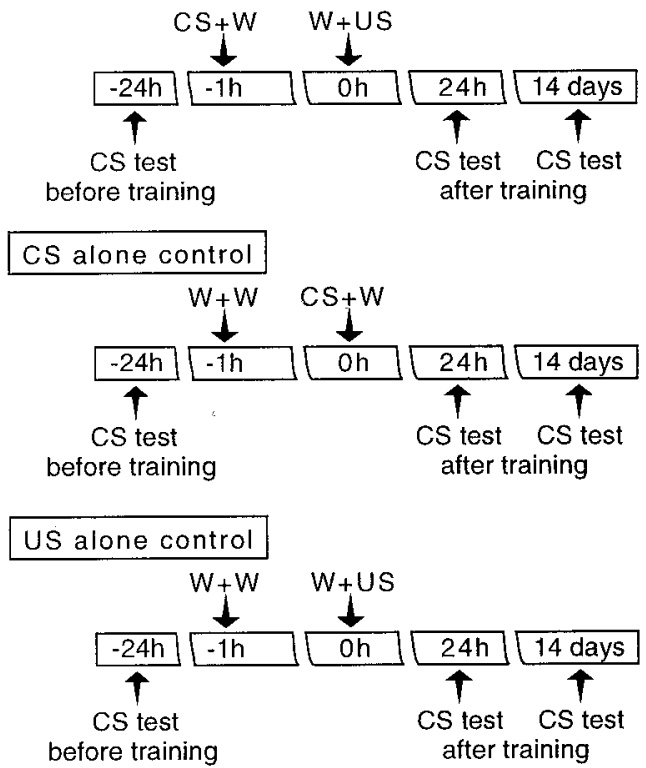

Bi

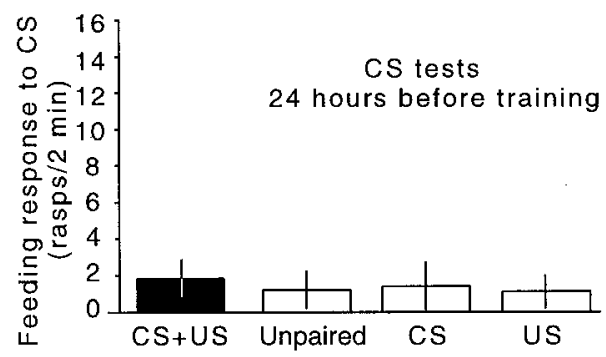

Bii

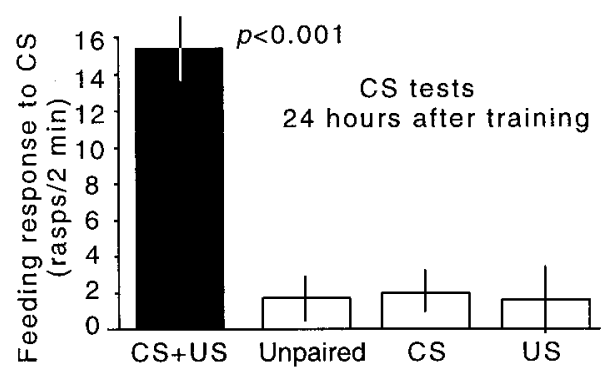

Biii

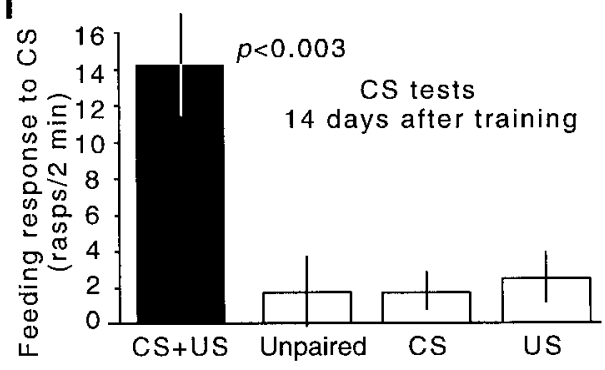

Figure 3. One-trial chemical conditioning of the feeding response results in LTM formation in Lymnaea. A, All snails were tested for feeding responses in the presence of water (a disturbance factor) and amyl-acetate (the CS) $24 \mathrm{hr}$ before training. For each animal, an amyl-acetate minus water response score was generated, and this was used to quantify the feeding response to the CS. The pretested animals were divided into four groups matched for their mean feeding responses to the CS (Bi). Conditioned snails (black bar) were subjected to a single paired CS + US trial (amyl-acetate paired with sucrose), whereas control snails (white bars) were subjected to an unpaired CS and US trial (Unpaired control) or CS or US alone control trials. Water $(W)$ was used to control for volume changes associated with the addition of the CS or US (a disturbance factor). After each trial, the animals were rinsed and transferred back to their home tank. All four groups were retested for the CS $24 \mathrm{hr}$ and $14 \mathrm{~d}$ after training. B, Conditioned animals (black bars) show significantly stronger feeding responses to the CS than controls (white bars), at both $24 \mathrm{hr}$ (Bii) and $14 \mathrm{~d}$ (Biii) after training. Only the trained group shows a significant increase in the response to the CS (Bii, Biii) compared with pretraining levels $(\mathrm{Bi})$. The feeding responses to the CS data are shown as means \pm SE. The groups showed no differences in their pretraining feeding rates in water and amyl-acetate or in their post-training feeding rates in water (see Results). For details of the statistical analyses, also see Results.

levels $(2.8 \pm 1.2 \mathrm{rasps} / 2 \mathrm{~min})$ (ANOVA with repeated measures; before and after training, $F_{1,36}=3.3, p=0.08$; training, $F_{1,36}=$ $1.5, p=0.1$ ). Thus for the animals injected at $4 \mathrm{hr}$ after training, there is no associative LTM (Fig. 5Bii). In contrast, conditioned snails $(n=20)$ injected with PTIO $6 \mathrm{hr}$ after training (Fig. 5Biii, black bars) did show associative LTM. This was first detected by an ANOVA with repeated measures (before and after training, $F_{1,37}=9.1, p<0.005$; training, $\left.F_{1,37}=7.1, p<0.01\right)$ and confirmed by finding a significant difference between posttraining and pretraining scores within the CS + US group $(6.8 \pm$ 1.4 vs $0.9 \pm 1.4 \mathrm{rasps} / 2 \mathrm{~min} ; p<0.001)$ and a significant difference between post-training scores in the CS + US and the unpaired control group $(n=19 ; 0.8 \pm 1.4 \mathrm{rasps} / 2 \mathrm{~min} ; p<0.002)$ (Fig. 5Biii).

Comparisons in all PTIO injection experiments that used both conditioned and unpaired control groups therefore showed that NO depletion beginning $10 \mathrm{~min}$ after training and up to $4 \mathrm{hr}$ impaired the formation of an associative LTM. By $6 \mathrm{hr}$ after training, however, PTIO does not interfere with LTM formation (Fig. 5Biii). These results, together with the experiment shown in
Figure $5 A$, provided very strong evidence that for up to $\sim 5 \mathrm{hr}$ after conditioning, NO was needed for the formation of associative LTM but not subsequently. Importantly, the experiments using later injection times also showed that there were no persistent indirect effects of PTIO (e.g., producing sick animals) that might reduce the conditioned response at test.

\section{The effect of NO depletion on LTM also can be detected in vitro}

Although a detailed cellular analysis of the role of NO in the formation of LTM was beyond the scope of this study, it was important to demonstrate that the effects of NO depletion on LTM formation can be recorded at both the behavioral and cellular level in the same animals. We achieved this by intracellular recording of the activity of an identified modulatory neuron, CV1a (McCrohan, 1984), and a motoneuron, B3 (Benjamin and Rose, 1979), in head-brain preparations of trained snails while applying the chemical CS to the lips (Fig. 6Bi). Both of these cell types have been used in the same type of preparation to monitor CS-evoked fictive feeding, a readout of the appetitive memory 
A

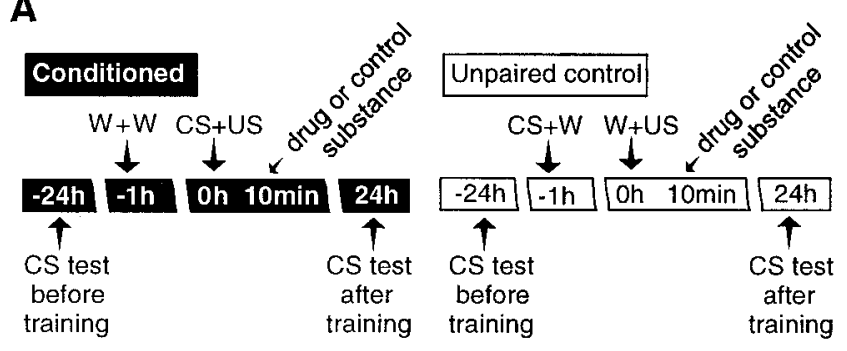

Bi

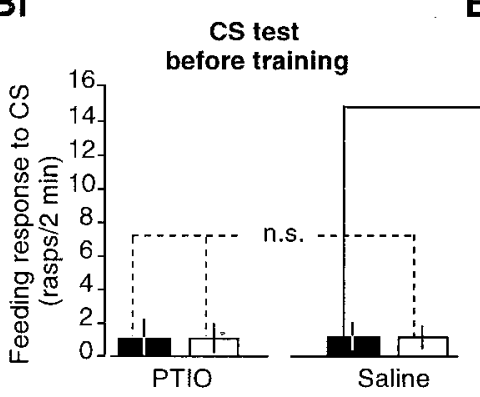

Bii

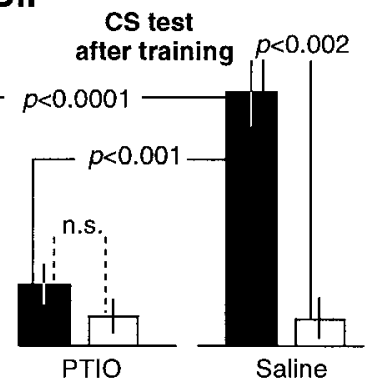

$\mathrm{Ci}$

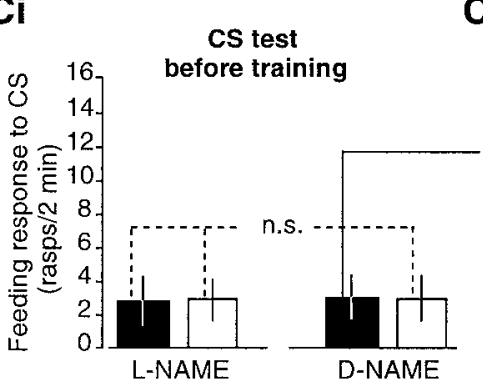

Cii

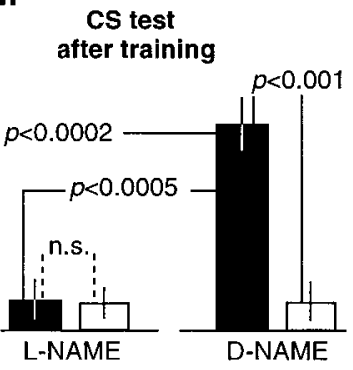

Di

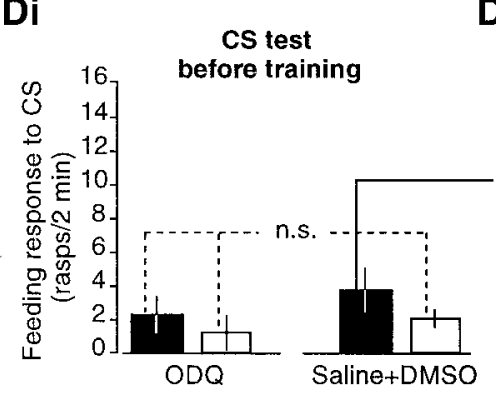

Dii

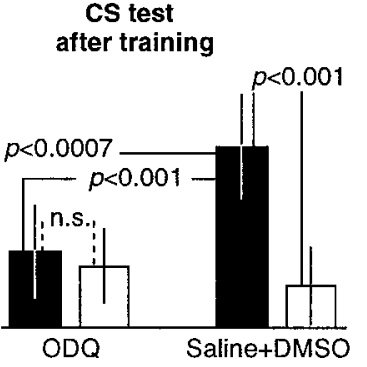

Figure 4. Blocking of the NO-cGMP pathway impairs LTM after singletrial appetitive classical conditioning. $A$, For each experiment shown in this figure, a group of snails were pretested for the CS $24 \mathrm{hr}$ before training and divided into four matched groups (pretraining response levels are shown in $B i, C i$, and $D i$, respectively). Conditioned snails (black bar) were subjected to one-trial CS + US training. Unpaired control snails (white bar) were subjected to a CS + water $(W)$ trial followed after $1 \mathrm{hr}$ by a water + US trial. Water was used to control for volume changes associated with the addition of the CS or US (control groups), or both (conditioned groups). At the end of each trial, the animals were rinsed and transferred back to their home tank. Ten minutes after the training or control trial, animals were injected with NO-cGMP pathway blocking drugs or control substances. Both conditioned and control snails were tested for feeding response to the CS $24 \mathrm{hr}$ after the training trial. $B$, The effect of NO depletion after training. There is no difference in PTIOinjected conditioned snails (Bii, PTIO, black bar) compared with the PTIO-injected unpaired controls (Bii, PTIO, white bar). In contrast, vehicle-injected conditioned snails (Bii, Saline, black bar) show significantly higher feeding responses to the CS than vehicle-injected unpaired trace at the electrophysiological level (Whelan and McCrohan, 1996; Kemenes et al., 1997; Staras et al., 1998, 1999). The preparations made from conditioned animals were injected with PTIO or saline just after training and tested behaviorally before dissection (Fig. 5Ai). As expected, our behavioral experiments revealed a strong inhibition of LTM formation by PTIO, but not saline, and showed no difference between controls and PTIOinjected trained animals. Furthermore, it has already been demonstrated that semi-intact preparations from control snails show no fictive feeding responses to the chemical CS (Whelan and McCrohan, 1996). Therefore, our electrophysiological analysis concentrated on the PTIO and saline-injected trained groups because they provide the most direct insight into the effect of NO depletion on the electrophysiological expression of LTM at the cellular level.

Preparations from PTIO-injected conditioned snails $(n=5)$ showed fictive feeding responses $(-0.8 \pm 0.8$ cycles $/ 2 \mathrm{~min})$ that were significantly weaker $(p<0.01)$ than those from salineinjected conditioned snails $(5.4 \pm 1.8$ cycles $/ 2 \mathrm{~min} ; n=5)$ (Fig. 6 Aii). This closely reflected the significant behavioral differences that were found between both the same two groups of animals before dissection (Fig. 6 $\mathrm{Ai}$ ) and the two larger groups of animals from which the electrophysiologically tested animals were randomly derived (PTIO, $14.2 \pm 2.1 \mathrm{rasps} / 2 \mathrm{~min}, n=15$; saline, $4.6 \pm 1.2 \mathrm{rasps} / 2 \mathrm{~min} ; n=14 ; p<0.01$; data not shown in Fig. $6 A$ ). Figure $6 B i i$ shows an electrophysiological recording from one of the five saline-injected conditioned snail preparations that were used for the above quantitative analysis. This preparation, like the others in the same group, responded to the CS by producing a series of fictive feeding cycles, each consisting of clearly identifiable sequences of N1 (protraction), N2 (rasp), and N3 (swallow) phase synaptic inputs from the feeding central pattern generator (CPG) (Fig. 6Bii, expanded time-base trace). In the $\mathrm{B} 3$ motoneuron, $\mathrm{CPG}$ inputs are known to produce one phase of inhibition (N1) followed by two phases of excitation (N2 and N3), whereas in the CV1a modulatory neuron they first produce strong excitation (N1), followed by two phases of inhibition (N2 and N3) (Benjamin and Elliott, 1989), and therefore the occurrence of these characteristic sequences in both cell types was used as a specific criterion for identifying each cycle. By

\footnotetext{
controls (Bii, Saline, white bar). $C$, The effect of inhibiting NOS after training. Conditioned L-NAME injected snails (Cii, L-NAME, black bar) show no increase in their post-training feeding responses to the $C S$, which are statistically similar to L-NAME-injected unpaired controls (Cii, $L-N A M E$, white bar). The feeding response to the CS in the D-NAMEinjected conditioned snails (Cii, D-NAME, black bar) is significantly higher than the response in both D-NAME-injected unpaired controls (Cii, $D-N A M E$, white bar) and L-NAME-injected conditioned animals. $D$, The effect of inhibiting sGC after training. Conditioned ODQ-injected snails (Dii, $O D Q$, black bar) show no increase in their post-training feeding responses to the CS, which are statistically similar to ODQ-injected unpaired controls (Dii, ODQ, white bar). The feeding response to the CS in the saline + DMSO-injected conditioned snails (Dii, Saline+DMSO, black bar) is significantly higher than the response in both saline + DMSO-injected unpaired controls (Dii, Saline $+D M S O$, white bar) and ODQ-injected conditioned animals. In all three experiments, only conditioned snails injected with control substances show post-training responses to the CS that are significantly stronger than pretraining response levels $(B i, C i, D i$, black bars $)$. All data in this figure are shown as means \pm SE. In each experiment, the groups showed no differences in their pretraining feeding rates in water and amyl-acetate or in their posttraining feeding rates in water (see Results). For details of the statistical analyses, also see Results.
} 
Ai

Conditioned

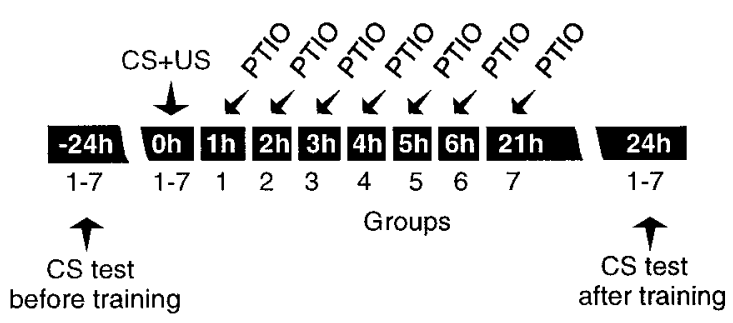

Aii

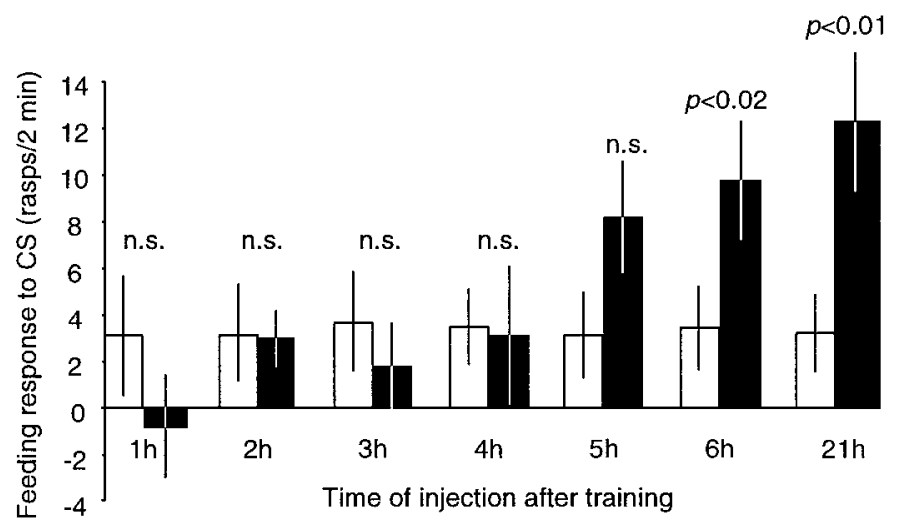

Bii

Bi

Conditioned
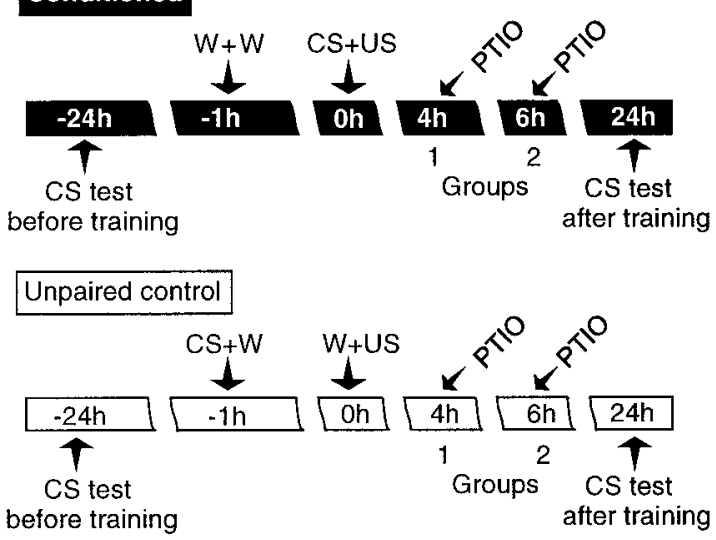

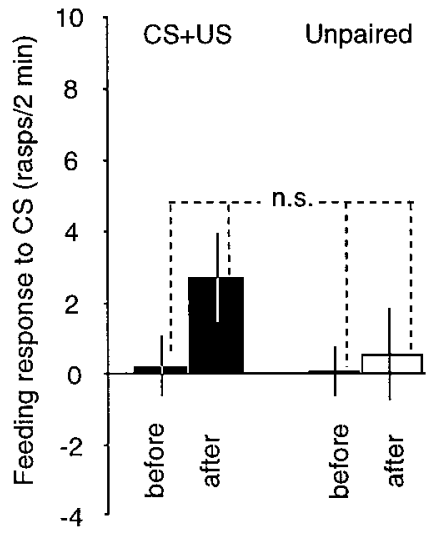

Group 1: 4 h postGroup 1: 4 h post-
training injection
Biii

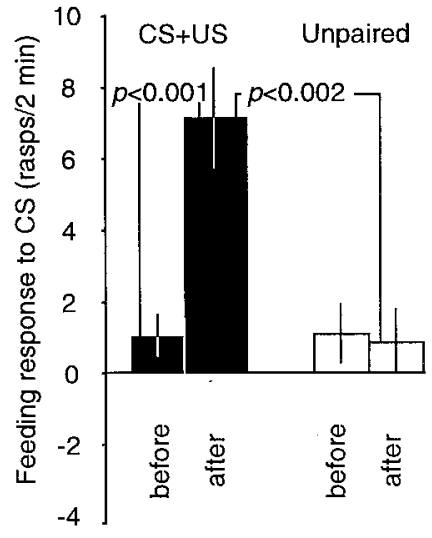

Figure 5. Critical time window for the impairment of LTM by PTIO-induced depletion of NO. Ai, A single large group of animals were initially tested for feeding response to the CS (white bar, $-24 \mathrm{hr}$ ) before they were conditioned $24 \mathrm{hr}$ later $(0 h, C S+U S)$. The conditioned animals were divided into seven groups (matched for pretraining response to the CS), each injected with PTIO at one of seven different time points after training (Group 1, $1 h$; Group 2, 2h;. . .Group 7, 21h). All snails were retested for feeding response to the CS at $24 \mathrm{hr}$ after training. Ail, Depletion of NO by injection of PTIO up to $4 \mathrm{hr}$ after training completely prevents the conditioned feeding responses (black bars), which are the same as the feeding responses to the CS before training (white bars). After $>4 \mathrm{hr}$, the effect of NO depletion starts to decline, and after $>5 \mathrm{hr}$ after training, NO depletion no longer has an effect on the conditioned feeding responses to the CS, which are significantly greater than before training and greater than the post-training responses in the groups injected between 1 and $4 \mathrm{hr}$ after training. $p$ values indicate significant within-group differences in the response to the CS before and after training. $B i$, For comparisons at the most critical time points revealed by the results in $A i i$, both conditioned and unpaired control groups were used that were injected with PTIO either $4 \mathrm{hr}$ (Group 1) or $6 \mathrm{hr}$ (Group 2) after the training or control trial. Bii, Conditioned animals (black bars) injected with PTIO $4 \mathrm{hr}$ after training show feeding responses to the CS that are not different from either pretraining levels or post-training control levels. Biii, In contrast, conditioned animals (black bars) injected with PTIO $6 \mathrm{hr}$ after training show feeding responses to the CS that are significantly stronger than both pretraining levels and post-training control levels. Data are shown as means \pm SE. In each experiment shown in this figure, the groups showed no differences in their pretraining feeding rates in water and amyl-acetate or in their post-training feeding rates in water (see Results). For details of the statistical analyses, also see Results.

contrast, Figure 6Biii shows a recording from a PTIO-injected snail preparation that showed no fictive feeding response to the CS in CV1a and only a slight increase in non-CPG driven burst activity in B3. In the same preparation, fictive feeding could still be activated by artificially depolarizing CV1a (Fig. 6Biii), a modulatory neuron well known for its ability to drive the feeding CPG (McCrohan, 1984). Interestingly, a short-latency maintained depolarization of CV1a by the CS (Fig. 6Bii,Biii, arrow), seen in all preparations, was significantly weaker in preparations from conditioned snails injected with PTIO $(3.8 \pm 1.0 \mathrm{mV})$ than in preparations from conditioned and saline-injected snails (12.2 $\pm 2.8 \mathrm{mV} ; p<0.02$ ) (Fig. 6Ci). This difference was not caused by a conditioning-related change in CV1a resting potential, which was not different in the two groups (Fig. 6Cii). The presence of a depolarizing response to the CS in CV1a has been also described in preparations derived from chemically conditioned uninjected Lymnaea, and it was found to be stronger than in preparations derived from control animals (Whelan and McCrohan, 1996).

\section{DISCUSSION}

Here we have shown that consolidation of a long-term associative memory trace in Lymnaea requires an intact NO-cGMP signaling pathway. Depletion of NO and inhibition of NOS or soluble guanylyl cyclase after acquisition in a single trial resulted in impaired memory. Our time course experiments revealed a posttraining period of $\sim 5 \mathrm{hr}$, during which there is an obligatory requirement for NO for LTM formation. This is followed by a fairly sharp transition period between 5 and $6 \mathrm{hr}$, after which inhibition of NO signaling does not impair LTM formation. It is 
Ai

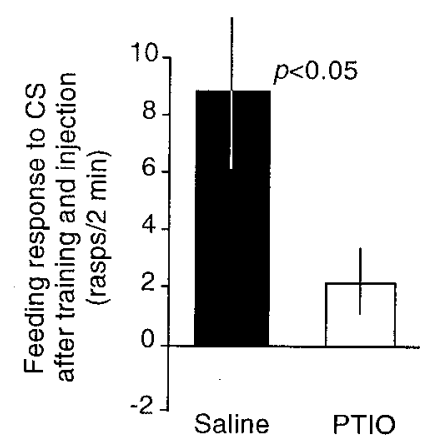

Aii

$\mathrm{Bi}$

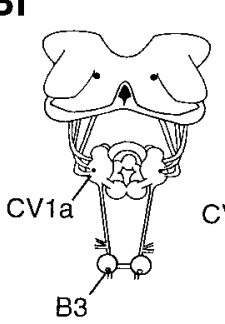

Bii Saline injected
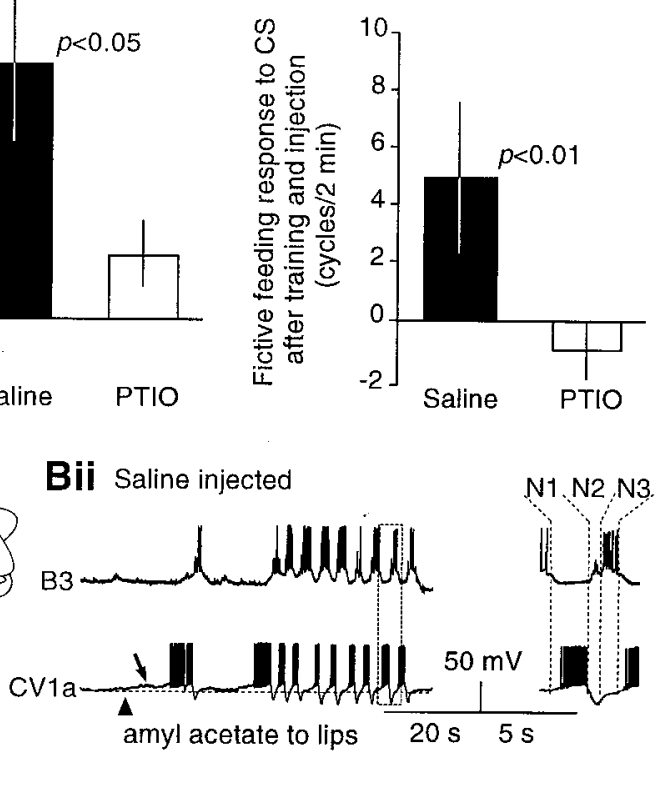

Biii PTIO injected

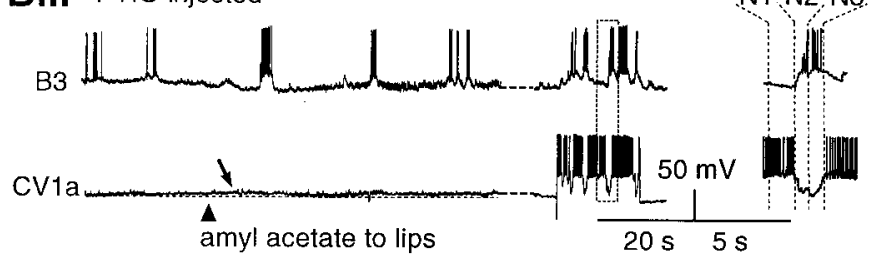

Ci

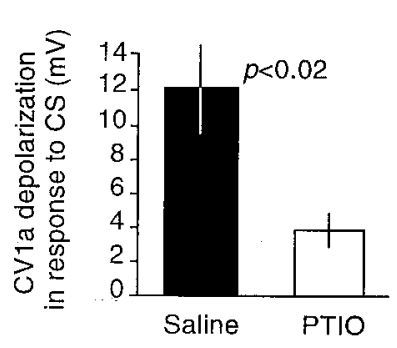

Cii

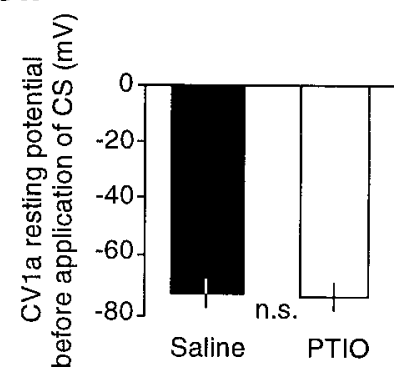

Figure 6. The behavioral effect of PTIO-induced NO depletion on LTM is amenable to a cellular analysis. $A$, The same group of conditioned and saline-injected snails (black bars) shows both a behavioral ( $A i$, feeding) and an electrophysiological (Aii, fictive feeding) response to the CS, and these are both significantly stronger than corresponding response levels in conditioned snails that were subsequently injected with PTIO. $B i$, The semi-intact preparation used in this experiment. The positions of the two types of neurons that were recorded are shown in the cerebral ganglia (CV1a, a modulatory neuron) and the buccal ganglia (B3, a motoneuron). The cerebral ganglia are connected to the lips by the lip and tentacle nerves. Bii, A typical preparation from one of the five saline-injected conditioned animals that were used in the quantitative analysis shown in Aii. Both CV1a and B3 show a series of fictive feeding cycles in response to amyl-acetate applied to the lips (start of application indicated by arrowhead). The characteristic N1/protraction, N2/rasp, and N3/swallow phases of fictive feeding (Benjamin and Elliott, 1989) are marked on an expanded time-base trace of a section with one of the cycles (boxed). Biii, A typical preparation from one of the five PTIO-injected conditioned animals that were used in the quantitative analysis shown in Aii. Neither CV1a nor B3 shows fictive feeding cycles in response to amyl-acetate (start of application indicated by arrowhead). However, activation of CV1a by injection of a steady depolarizing current can still drive activity difficult to estimate precisely how sharp this transition is, because although the effect of a single injection with PTIO is transient, it can last for up to $1 \mathrm{hr}$. Nevertheless, our experiments suggest that $\mathrm{NO}$ is required continuously for the $5 \mathrm{hr}$ time window; that is, suppression of LTM can be achieved by transient inhibition of NO signaling at any time during that period.

Most previous behavioral studies demonstrated a role for the NO-cGMP pathway during the acquisition phase of learning in vertebrates and invertebrates. For example, NO was shown to be involved in the acquisition of olfactory learning in sheep (Kendrick et al., 1997) and a snail, Helix (Teyke, 1996), and touch learning in octopuses (Robertson et al., 1994). However, our results provide important new information on its role as a factor contributing to processes of memory consolidation starting immediately after the acquisition phase of learning. The exact nature of this contribution is unknown, but it is tempting to speculate that it is based on NO being required for the gradual buildup of a biochemical process that lasts for $\sim 5 \mathrm{hr}$ and must reach a threshold level before the memory trace can be consolidated. The interruption of this hypothetical process at any time during the critical time window therefore would both prevent the process from reaching threshold and block LTM formation. Although the evidence provided by our experiments strongly favors the conclusion that appetitive LTM in Lymnaea has a direct NO-cGMP dependence in this critical time window after conditioning, an alternative explanation for our results may be that pharmacological suppression of the NO-cGMP pathway causes some transient physiological effects that may only indirectly disrupt the memory consolidation process. Thus, the process of consolidation itself may involve a completely different pathway. However, several recent studies in both vertebrates ( $\mathrm{Lu}$ et al., 1999) and invertebrates (Lewin and Walters, 1999; Müller, 2000) have already directly implicated the NO-cGMP pathway in memory formation, and our new data appear to extend these studies. Whatever the mechanism of the NO-cGMP dependence of LTM formation in Lymnaea, its elucidation will depend on being able to investigate these behavioral observations at a cellular and molecular level in the future.

One-trial avoidance and odor preference learning in chicks, mice, and rats have been shown previously to depend on NO synthesis (Holscher and Rose, 1993; Baratti and Kopf, 1996; Rickard et al., 1998; Samama and Boehm, 1999). Our study, however, is the first demonstration that single-trial appetitive classical conditioning can lead to NO-dependent LTM formation. In contrast, in the honeybee, NO is apparently not required for the formation of one type of persistent associative memory after single-trial olfactory classical conditioning (Müller, 1996). Interestingly, however, preventing the production of NO during multitrial conditioning in the honeybee does impair a more robust form of long-term memory (Müller, 1996). Moreover, even a

$\leftarrow$

in the feeding CPG, which can be monitored as N1, N2, and N3 phase synaptic inputs on both CV1a and B3 (expanded time-base trace of boxed section). $\mathrm{Ci}$, The depolarization produced in $\mathrm{CV} 1 \mathrm{a}$ in response to amylacetate (Bii, Biii, arrows) is significantly stronger in preparations derived from saline-injected animals (Saline, black bar) than in those obtained from PTIO-injected conditioned animals (PTIO, white bar). Cii, The resting potential levels of CV1a are virtually identical in the two groups, indicating that the difference in the depolarizing response is not caused by a difference in CV1a membrane potential between the two types of preparation. All data in this figure are shown as means \pm SE. For details of the statistical analyses, see Results. 
single conditioning trial in the bee can lead to similarly robust LTM if caged cGMP is photoreleased in the antennal lobes after training (Müller, 2000). Further similarity between the two systems is indicated by the dependence of LTM on protein synthesis (Wüstenberg et al., 1998; I. Kemenes et al., 2000). It is therefore conceivable that some key molecular mechanisms linking NO to protein synthesis-dependent processes underlying LTM formation are shared by these two systems and possibly by others as well. Studies in mice showed that NO contributes to late-phase LTP by stimulating sGC- and cGMP-dependent protein kinase (PKG), which acts in parallel with cAMP-dependent protein kinase (PKA) to increase phosphorylation of the transcription factor CREB (Lu et al., 1999). Evidence from sensory neurons in Aplysia showed that the induction of at least one form of nonassociative LTM, long-term hyperexcitability after a noxious stimulus, depends on transcriptionally dependent CREB-mediated effects of the NO-cGMP-PKG pathway, but not on PKA (Lewin and Walters, 1999). In the bee, as in mice, a link between the NO-cGMP pathway and PKA- and protein synthesis-dependent associative LTM has been reported (Müller, 2000). The mice, bee, and Aplysia results support the notion that the NO-cGMPPKG pathway directly regulates gene transcription during the formation of various types of LTM. This is also likely to be the case in Lymnaea, where a link between NO-mediated and CREBdependent mechanisms of appetitive LTM formation has yet to be established.

Concerning the duration of the NO requirement after training in other systems, experiments in mice show memory impairment caused by the injection of L-NAME up to $3 \mathrm{hr}$ after training (Baratti and Kopf, 1996). Using another approach, Telegdy and Kokavszky (1997) showed that administration of L-arginine (a rate-limiting substrate for NO production by NOS) immediately after passive avoidance learning in rats improved the consolidation of memory for $\sim 6 \mathrm{hr}$. Additional evidence for a post-training role for NO was found in the rat hippocampus, where NOS activity increased by $45 \%$ immediately after avoidance training, and inhibition of NOS immediately after training caused impairment of memory (Bernabeu et al., 1995).

All of the examples cited above provide compelling evidence that NO plays a crucial role in the early post-training phase of memory formation in both vertebrate and invertebrate systems. This again indicates conservation of underlying mechanisms. This is important because the feeding system in Lymnaea provides an opportunity to identify cellular and molecular processes likely to be affected by NO. A notable advantage of the feeding system in Lymnaea is that the neurons of the feeding CPG and their synaptic connectivity have been described, and a number of modulatory influences have been identified (for review, see Benjamin et al., 2000). This will greatly facilitate the identification of NO-dependent cellular and molecular mechanisms involved in LTM formation. It is known, for example, that NO mediates sucrose-induced activation of the feeding CPG (Moroz et al., 1993; Elphick et al., 1995), but importantly, NO is not involved directly in the generation of the feeding pattern by the interneurons of the CPG (Elphick et al., 1995). This indicates that the focus of our further investigation of NO-dependent plasticity in LTM should be directed at the various modulatory influences on the CPG that have been characterized. The cerebral giant cells are one identified neuron type that can modulate the feeding CPG (McCrohan and Audesirk, 1987; Yeoman et al., 1996). These have been implicated in learning (G. Kemenes et al., 2000) and are known to express the neuronal NOS gene (Korneev et al.,
1998). Another important modulatory cell type is the paired CV1a neurons, which are capable of driving activity in the feeding CPG (McCrohan, 1984) and modulating feeding motor output (Kemenes et al., 2001). Our electrophysiological tests showed that the CS activated significantly weaker fictive feeding rhythms in both CV1a and a motoneuron, B3, in semi-intact preparations made from PTIO-injected versus saline-injected snails. CS-evoked fictive feeding is an electrophysiological readout of the appetitive memory trace in the snail feeding system (Whelan and McCrohan, 1996; Kemenes et al., 1997; Staras et al., 1998, 1999), and therefore this result demonstrates that the effect of NO depletion on LTM is amenable to analyses at the cellular level. The observation that a short-latency maintained excitatory input to CV1a from the CS pathway in preparations from conditioned animals was weaker after post-training treatment with PTIO indicates that a potential target for NO-dependent plasticity may be located in the afferent pathways of the feeding system.

In conclusion, our study not only identifies a critical time window of NO dependence of associative LTM after classical conditioning of the feeding response in Lymnaea, but also has important implications for our understanding of fundamental cellular and molecular processes underlying memory formation.

\section{REFERENCES}

Akaike T, Yoshida M, Miyamoto Y, Sato K, Kohno M, Sasamoto K, Miyazaki K, Ueda S, Maeda H (1993) Antagonistic action of imidazolineoxyl $N$-oxides against endothelium-derived relaxing factor/NO through a radical reaction. Biochemistry 32:827-832.

Alexander Jr J, Audesirk TE, Audesirk GJ (1984) One-trial reward learning in the snail Lymnaea stagnalis. J Neurobiol 15:67-72.

Baratti CM, Kopf SR (1996) A nitric oxide synthase inhibitor impairs memory storage in mice. Neurobiol Learn Mem 65:197-201.

Benjamin PR, Elliott CJH (1989) Snail feeding oscillator: the central pattern generator and its control by modulatory interneurons. In: Neuronal and cellular oscillators (Jacklet J, ed), pp 173-214. New York: Dekker.

Benjamin PR, Rose RM (1979) Central generation of bursting in the feeding system of the snail Lymnaea stagnalis. J Exp Biol 80:93-118.

Benjamin PR, Winlow W (1981) The distribution of three wide-acting synaptic inputs to identified neurons in the isolated brain of Lymnaea. Comp Biochem Physiol [A] 70:293-307.

Benjamin PR, Staras K, Kemenes G (2000) A systems approach to the cellular analysis of associative learning in the pond snail Lymnaea. Learn Mem 7:124-131.

Bernabeu R, de Stein ML, Fin C, Izquierdo I, Medina JH (1995) Role of hippocampal NO in the acquisition and consolidation of inhibitory avoidance learning. NeuroReport 6:1498-1500.

Blokland A, de Vente J, Prickaerts J, Honig W, Markerink-van Ittersum M, Steinbusch H (1999) Local inhibition of hippocampal nitric oxide synthase does not impair place learning in the Morris water escape task in rats. Eur J Neurosci 11:223-232.

Elofsson R, Carlberg M, Moroz L, Nezlin L, Sakharov D (1993) Is nitric oxide (NO) produced by invertebrate neurones? NeuroReport 4:279-282.

Elphick MR, Kemenes G, Staras K, O'Shea M (1995) Behavioral role for nitric oxide in chemosensory activation of feeding in a mollusc. J Neurosci 15:7653-7664.

Fedele E, Raiteri M (1999) In vivo studies of the cerebral glutamate receptor/NO/cGMP pathway. Prog Neurobiol 58:89-120.

Floyd PD, Moroz LL, Gillette R, Sweedler JV (1998) Capillary electrophoresis analysis of nitric oxide synthase related metabolites in single identified neurons. Anal Chem 70:2243-2247.

Frisch C, Dere E, Silva MA, Godecke A, Schrader J, Huston JP (2000) Superior water maze performance and increase in fear-related behavior in the endothelial nitric oxide synthase-deficient mouse together with monoamine changes in cerebellum and ventral striatum. J Neurosci 20:6694-6700.

Garthwaite J, Southam E, Boulton CL, Nielsen EB, Schmidt K, Mayer B (1995) Potent and selective inhibition of nitric oxide-sensitive guanylyl cyclase by $1 \mathrm{H}-[1,2,4]$ oxadiazolo[4,3-a]quinoxalin-1-one. Mol Pharmacol 48:184-188

Gelperin A (1994) Nitric oxide mediates network oscillations of olfactory interneurons in a terrestrial mollusc. Nature 369:61-63.

Hawkins RD, Son H, Arancio O (1998) Nitric oxide as a retrograde messenger during long-term potentiation in hippocampus. Prog Brain Res 118:155-172. 
Holscher C, Rose SP (1993) Inhibiting synthesis of the putative retrograde messenger nitric oxide results in amnesia in a passive avoidance task in the chick. Brain Res 619:189-194.

Jacklet JW (1997) Nitric oxide signaling in invertebrates. Invert Neurosci 3:1-14.

Jones NG, Kemenes G, Benjamin PR (2001) Selective expression of electrical correlates of differential appetitive classical conditioning in a feeding network. J Neurophysiol 85:89-97.

Kemenes G, Elliott CJH, Benjamin PR (1986) Chemical and tactile inputs to the Lymnaea feeding system: effects on behaviour and neural circuitry. J Exp Biol 122:113-137.

Kemenes G, Staras K, Benjamin PR (1997) In vitro appetitive classical conditioning of the feeding response in the pond snail Lymnaea stagnalis. J Neurophysiol 78:2351-2362.

Kemenes G, Jones NG, Staras K, Kemenes I, O’Shea M, Benjamin PR (2000) Cellular changes in the feeding system after tactile and chemical appetitive classical conditioning in Lymnaea. Eur J Neurosci 12:93.

Kemenes G, Staras K, Benjamin PR (2001) Multiple types of control by identified interneurons in a sensory-activated rhythmic motor pattern. J Neurosci 21:2903-2911.

Kemenes I, Kemenes G, Andrew RJ, Benjamin PR, O'Shea M (2000) Interruptions of the NO-cGMP pathway and protein synthesis block long-term memory formation after one-trial appetitive conditioning in Lymnaea. Eur J Neurosci 12:93.

Kendrick KM, Guevara-Guzman R, Zorrilla J, Hinton MR, Broad KD, Mimmack M, Ohkura S (1997) Formation of olfactory memories mediated by nitric oxide. Nature 388:670-674.

Kobayashi S, Sadamoto H, Ogawa H, Kitamura Y, Oka K, Tanishita K, Ito $\mathrm{E}$ (2000) Nitric oxide generation around buccal ganglia accompanying feeding behavior in the pond snail, Lymnaea stagnalis. Neurosci Res 38:27-34.

Koh HY, Jacklet JW (1999) Nitric oxide stimulates cGMP production and mimics synaptic responses in metacerebral neurons of Aplysia. J Neurosci 19:3818-3826.

Koh HY, Jacklet JW (2001) Nitric oxide induces cGMP immunoreactivity and modulates membrane conductance in identified central neurons of Aplysia. Eur J Neurosci 3:553-560.

Kojima S, Nanakamura H, Nagayama S, Fujito Y, Ito E (1997) Enhancement of an inhibitory input to the feeding central pattern generator in Lymnaea stagnalis during conditioned taste-aversion learning. Neurosci Lett 230:179-182.

Korneev SA, Piper MR, Picot J, Phillips R, Korneeva EI, O'Shea M (1998) Molecular characterization of NOS in a mollusc: expression in a giant modulatory neuron. J Neurobiol 35:65-76.

Lewin MR, Walters ET (1999) Cyclic GMP pathway is critical for inducing long-term sensitization of nociceptive sensory neurons. Nat Neurosci 1:18-23.

Lu YF, Kandel ER, Hawkins RD (1999) Nitric oxide signaling contributes to late-phase LTP and CREB phosphorylation in the hippocampus. J Neurosci 19:10250-10261.

McCrohan CR (1984) Initiation of feeding motor output by an identified interneurone in the snail Lymnaea stagnalis. J Exp Biol 113:351-366.

McCrohan CR, Audesirk TE (1987) Initiation, maintenance and modification of patterned buccal motor output by the cerebral giant cells of Lymnaea stagnalis. Comp Biochem Physiol 87A:969-977.

Moroz LL, Park JH, Winlow W (1993) Nitric oxide activates buccal motor patterns in Lymnaea stagnalis. NeuroReport 4:643-646.

Moroz LL, Winlow W, Turner RW, Bulloch AG, Lukowiak K, Syed NI
(1994) Nitric oxide synthase-immunoreactive cells in the CNS and periphery of Lymnaea. NeuroReport 5:1277-1280.

Müller U (1996) Inhibition of nitric oxide synthase impairs a distinct form of long-term memory in the honeybee, Apis mellifera. Neuron 16:541-549.

Müller U (1997) The nitric oxide system in insects. Prog Neurobiol 51:363-381.

Müller U (2000) Prolonged activation of cAMP-dependent protein kinase during conditioning induces long-term memory in honeybees. Neuron 27:159-168.

Ogunshola O, Picot J, Piper M, Korneev S, O'Shea M (1995) Molecular analysis of the NO-cGMP signalling pathway in insect and molluscan CNS. Soc Neurosci Abstr 21:631.

Park JH, Straub VA, O'Shea M (1998) Anterograde signaling by nitric oxide: characterization and in vitro reconstitution of an identified nitrergic synapse. J Neurosci 18:5463-5476.

Rickard NS, Ng KT, Gibbs ME (1998) Further support for nitric oxidedependent memory processing in the day-old chick. Neurobiol Learn Mem 69:79-86.

Robertson JD, Bonaventura J, Kohm AP (1994) Nitric oxide is required for tactile learning in Octopus vulgaris. Proc R Soc Lond B Biol Sci 256:269-273.

Rose SP (2000) God's organism? The chick as a model system for memory studies. Learn Mem 7:1-17.

Samama B, Boehm N (1999) Inhibition of nitric oxide synthase impairs early olfactory associative learning in newborn rats. Neurobiol Learn Mem 71:219-231.

Sattelle DB, Lane NJ (1972) Architecture of gastropod central nervous tissues in relation to ionic movements. Tissue Cell 4:253-270.

Schweighofer N, Ferriol G (2000) Diffusion of nitric oxide can facilitate cerebellar learning: a simulation study. Proc Natl Acad Sci USA 97:10661-10665.

Serfözö Z, Elekes K, Varga V (1998) NADPH-diaphorase activity in the nervous system of the embryonic and juvenile pond snail, Lymnaea stagnalis. Cell Tissue Res 292:579-586.

Staras K, Kemenes G, Benjamin PR (1998) Neurophysiological correlates of unconditioned and conditioned feeding behavior in the pond snail Lymnaea stagnalis. J Neurophysiol 79:3030-3040.

Staras K, Kemenes G, Benjamin PR (1999) Cellular traces of behavioral classical conditioning can be recorded at several specific sites in a simple nervous system. J Neurosci 19:347-357.

Telegdy G, Kokavszky R (1997) The role of nitric oxide in passive avoidance learning. Neuropharmacology 36:1583-1587.

Teyke T (1996) Nitric oxide, but not serotonin, is involved in acquisition of food-attraction conditioning in the snail Helix pomatia. Neurosci Lett 206:29-32.

Van Wagenen S, Rehder V (1999) Regulation of neuronal growth cone filopodia by nitric oxide. J Neurobiol 2:168-185.

Whelan HA, McCrohan CR (1996) Food-related conditioning and neuronal correlates in the freshwater snail Lymnaea stagnalis. J Molluscan Stud 62:483-494.

Wüstenberg D, Gerber B, Menzel R (1998) Long- but not medium-term retention of olfactory memories in honeybees is impaired by actinomycin-D and anisomycin. Eur J Neurosci 10:2742-2745.

Yeoman MS, Brierley MJ, Benjamin PR (1996) Central pattern generator interneurons are targets for the modulatory serotonergic cerebral giant cells in the feeding system of Lymnaea. J Neurophysiol 75:11-25. 